\title{
Homoclinic solutions for $n$-dimensional $p$-Laplacian neutral differential systems with a time-varying delay
}

Fang Gao ${ }^{1}$ and Wenbin Chen ${ }^{2 *}$

"Correspondence:

cwb210168@126.com

${ }^{2}$ School of Automation, Nanjing

University of Science \& Technology, Nanjing, China

Full list of author information is

available at the end of the article

\begin{abstract}
In this paper, we investigate the existence of a set with $2 k T$-periodic solutions for $n$-dimensional $p$-Laplacian neutral differential systems with a time-varying delay $\left(\varphi_{p}(u(t)-C u(t-\tau))^{\prime}\right)^{\prime}+\frac{d}{d t} \nabla F(u(t))+G(u(t-\gamma(t)))=e_{k}(t)$ based on the coincidence degree theory of Mawhin. Combining this with the conclusion about uniform convergence and limit, we obtain the corresponding results on the existence of homoclinic solutions.
\end{abstract}

Keywords: Homoclinic solutions; Coincidence degree theory; Periodic solutions; Delay

\section{Introduction}

This paper focuses on the existence of homoclinic solutions for $n$-dimensional $p$-Laplacian neutral differential systems with a time-varying delay of the following form:

$$
\left(\varphi_{p}(u(t)-C u(t-\tau))^{\prime}\right)^{\prime}+\frac{d}{d t} \nabla F(u(t))+G(u(t-\gamma(t)))=e(t),
$$

where $p \in(1,+\infty), \varphi_{p}: \mathbb{R}^{n} \rightarrow \mathbb{R}^{n}, \varphi_{p}(u)=\left(\left|u_{1}\right|^{p-2} u_{1},\left|u_{2}\right|^{p-2} u_{2}, \ldots,\left|u_{n}\right|^{p-2} u_{n}\right)$ for $u \neq \mathbf{0}=$ $(0,0, \ldots, 0), F \in C^{2}\left(\mathbb{R}^{n}, \mathbb{R}\right), G \in C\left(\mathbb{R}^{n}, \mathbb{R}^{n}\right), e \in C\left(\mathbb{R}, \mathbb{R}^{n}\right), C=\operatorname{diag}\left(c_{1}, c_{2}, \ldots, c_{n}\right),\left|c_{i}\right| \neq 1(i=$ $1,2, \ldots, n), \tau$ and $T>0$ are given constants, $\gamma \in(\mathbb{R}, \mathbb{R}), \gamma(t+T)=\gamma(t)$ with $\gamma(t) \geq 0$.

In the past few decades, the existence of homoclinic solutions for second-order differential equations has been widely investigated by using critical point theory, the methods of bifurcation theory, or Mawhin's continuation theorem (see [1-8]). However, the corresponding results on the existence of homoclinic solutions to a neutral differential equation are relatively infrequent. For example, the existence of homoclinic solutions to a kind of second-order neutral functional differential systems was considered in [9]:

$$
\left((u(t)-C u(t-\tau))^{\prime \prime}+\frac{d}{d t} \nabla F(u(t))+G(u(t))+H(u(t-\gamma(t)))=e(t)\right.
$$

where $C=\left[c_{i j}\right]_{n \times n}$ is a real constant symmetric matrix, $F \in C^{2}\left(\mathbb{R}^{n}, \mathbb{R}\right), G, H \in C^{1}\left(\mathbb{R}^{n}, \mathbb{R}\right)$, $e \in C\left(\mathbb{R}, \mathbb{R}^{n}\right), \gamma \in(\mathbb{R}, \mathbb{R}), \gamma(t+T)=\gamma(t)$ with $\gamma(t) \geq 0$ and given constant $T>0$. Mean-

(c) The Author(s) 2018. This article is distributed under the terms of the Creative Commons Attribution 4.0 International License (http://creativecommons.org/licenses/by/4.0/), which permits unrestricted use, distribution, and reproduction in any medium, provided you give appropriate credit to the original author(s) and the source, provide a link to the Creative Commons license, and indicate if changes were made. 
while, $\mathrm{Du}[10]$ discussed the system

$$
(u(t)-C u(t-\tau))^{\prime \prime}+\frac{d}{d t} \nabla F(u(t))+\nabla G(u(t))=e(t)
$$

where $F \in C^{2}\left(\mathbb{R}^{n}, \mathbb{R}\right), G \in C^{1}\left(\mathbb{R}^{n}, \mathbb{R}\right) . e \in C\left(\mathbb{R}, \mathbb{R}^{n}\right), C=\operatorname{diag}\left(c_{1}, c_{2}, \ldots, c_{n}\right), c_{i}(i=1,2, \ldots, n)$ and $\tau$ are given constants. The existence of homoclinic solutions for Eq. (1.3) is obtained. Then Chen [11] studied the existence of homoclinic solutions for the class of neutral Duffing differential systems

$$
(u(t)-C u(t-\tau))^{\prime \prime}+\beta(t) x^{\prime}(t)+g(u(t-\gamma(t)))=p(t),
$$

where $\beta \in C^{1}(\mathbb{R}, \mathbb{R})$ with $\beta(t+T) \equiv \beta(t), g \in C\left(\mathbb{R}^{n}, \mathbb{R}^{n}\right), p \in C\left(\mathbb{R}, \mathbb{R}^{n}\right), \gamma \in(\mathbb{R}, \mathbb{R}), \gamma(t+T)=$ $\gamma(t)$ with $\gamma(t) \geq 0, T>0$ and $\tau$ are given constants; $\beta(t)$ is allowed to change sign, and $C=\left[c_{i j}\right]_{n \times n}$ is a constant symmetric matrix.

It is not hard to find that Eq. (1.1) can be converted to second-order neutral functional differential systems (1.2)-(1.4) when $p=2$. To our knowledge, there are few results reported in the literature regarding the existence of homoclinic solutions for $n$-dimensional $p$-Laplacian neutral differential systems with time-varying delay. Because of the term $\left(\varphi_{p}(u(t)-C u(t-\tau))^{\prime}\right)^{\prime}$ in Eq. (1.1), the method of Lemma 2.5 in [12] cannot be applied directly to prove that $\left|u_{0}^{\prime}(t)\right| \rightarrow 0$ as $|t| \rightarrow+\infty$. In this paper, we solve this problem by combining the conclusion about uniform convergence and Lemma 2.3 in [13].

Similarly to [9-11], we obtain the existence of a homoclinic solution for the equation by taking a series of the $2 k T$-periodic limit for the following equation:

$$
\left(\varphi_{p}(u(t)-C u(t-\tau))^{\prime}\right)^{\prime}+\frac{d}{d t} \nabla F(u(t))+G(u(t-\gamma(t)))=e_{k}(t)
$$

where $k \in \mathbb{N}$, and $e_{k}: \mathbb{R} \rightarrow \mathbb{R}^{n}$ is a $2 k T$-periodic function such that

$$
e_{k}(t)= \begin{cases}e(t), & t \in\left[-k T, k T-\varepsilon_{0}\right), \\ e\left(k T-\varepsilon_{0}\right)+\frac{e(-k T)-e\left(k T-\varepsilon_{0}\right)}{\varepsilon_{0}}\left(t-k T+\varepsilon_{0}\right), & t \in\left[k T-\varepsilon_{0}, k T\right],\end{cases}
$$

with a constant $\varepsilon_{0} \in(0, T)$ independent of $k$.

\section{Preliminaries}

Lemma 2.1 ([12]) If $u: \mathbb{R} \rightarrow \mathbb{R}^{n}$ is continuously differentiable on $\mathbb{R}, a>0, \mu>1$, and $p>1$ are constants, then for every $t \in \mathbb{R}$, we have the following inequality:

$$
|u(t)| \leq(2 a)^{-\frac{1}{\mu}}\left(\int_{t-a}^{t+a}|u(s)|^{\mu} d s\right)^{\frac{1}{\mu}}+a(2 a)^{-\frac{1}{p}}\left(\int_{t-a}^{t+a}\left|u^{\prime}(s)\right|^{p} d s\right)^{\frac{1}{p}} .
$$

Lemma 2.2 ([13]) Let $s \in C(\mathbb{R}, \mathbb{R})$ with $s(t+\omega) \equiv s(t)$ and $s(t) \in[0, \omega]$ for $t \in \mathbb{R}$. Suppose $p \in(1,+\infty),|s|_{0}=\max _{t \in[0, \omega]} s(t)$, and $u \in C^{1}(\mathbb{R}, \mathbb{R})$ with $u(t+\omega) \equiv u(t)$. Then

$$
\int_{0}^{\omega}|u(t)-u(t-s(t))|^{p} d t \leq|s|_{0}^{p} \int_{0}^{\omega}\left|u^{\prime}(t)\right|^{p} d t
$$


Lemma 2.3 ([14]) If $x \in(0,+\infty)$ satisfies the inequality $x^{s} \leq \alpha x^{q}+\beta x^{r}$ for some constants $s>q>r \geq 0, \alpha>0$, and $\beta>0$, then

$$
0<x \leq \inf _{\varepsilon \in(0,1)} \max \left\{\left(\frac{\beta}{\varepsilon}\right)^{\frac{1}{s-r}},\left(\frac{\alpha}{1-\varepsilon}\right)^{\frac{1}{s-q}}\right\} .
$$

Lemma 2.4 ([15]) Suppose $\tau \in C^{1}(\mathbb{R}, \mathbb{R})$ with $\tau(t+\omega) \equiv \tau(t)$ and $\tau^{\prime}(t)<1$ for $t \in[0, \omega]$. Then the function $t-\tau(t)$ has an inverse $\mu \in C(\mathbb{R}, \mathbb{R})$ such that $\mu(t+\omega) \equiv \mu(t)+\omega$ for $t \in \mathbb{R}$.

Lemma 2.5 ([16]) Suppose that $\Omega$ is an open bounded set in $X$ such that the following conditions are satisfied:

$\left[A_{1}\right]$ For each $\lambda \in(0,1)$, the equation

$$
\left(\varphi_{p}(u(t)-C u(t-\tau))^{\prime}\right)^{\prime}+\lambda \frac{d}{d t} \nabla F(u(t))+\lambda G(u(t-\gamma(t)))=\lambda e_{k}(t)
$$

has no solution on $\partial \Omega$.

$\left[A_{2}\right]$ The equation

$$
\triangle(a):=\frac{1}{2 k T} \int_{-k T}^{k T}\left[G(a)-e_{k}(t)\right] d t=0
$$

has no solution on $\partial \Omega \cap \mathbb{R}^{n}$.

$\left[A_{3}\right]$ The Brouwer degree

$$
d_{B}\left\{\triangle, \Omega \cap \mathbb{R}^{n}, 0\right\} \neq 0 .
$$

Then Eq. (1.5) has a 2kT-periodic solution in $\bar{\Omega}$.

Lemma 2.6 ([16]) Suppose that $c_{1}, c_{2}, \ldots, c_{n}$ are eigenvalues of a matrix $C$. If $\left|c_{i}\right| \neq 1(i=$ $1,2, \ldots, n)$, then $A$ has a continuous bounded inverse with the following properties:

(1) $\left\|A^{-1} f\right\| \leq\left(\sum_{i=1}^{n} \frac{1}{|1-| c_{i}||}\right)\|f\|$ for all $f \in C_{T}$,

(2) $\int_{0}^{T}\left|\left(A^{-1} f\right)(t)\right|^{p} d t \leq \alpha \int_{0}^{T}|f(t)|^{p} d t$ for all $f \in C_{T}$ and $p \geq 1$, where

$$
\alpha= \begin{cases}\max \left(\frac{1}{\left(1-\left|c_{i}\right|\right)^{2}}\right), & p=2, \\ \left(\sum_{i=1}^{n} \frac{1}{\left(1-\left|c_{i}\right| \frac{2 p}{2-p}\right.}\right)^{\frac{2-p}{2}}, & p \in[1,2), \\ \left(\sum_{i=1}^{n} \frac{1}{1-\left.\left|c_{i}\right|\right|^{q}}\right)^{\frac{p}{q}}, & p \in[2,+\infty),\end{cases}
$$

and $q$ is a constant such that $\frac{1}{p}+\frac{1}{q}=1$.

(3) $(A x)^{\prime}=A x^{\prime}$ for all $x \in C_{T}^{1}$.

Throughout this paper, for convenience, we list the following conditions and corresponding mathematical notation.

$\left[H_{1}\right]$ There are constants $m_{0}>0$ and $m_{1}>0$ such that

$$
\langle(E-C) x, G(x)\rangle \leq-m_{0}|x|^{p} \quad \text { for all } x \in \mathbb{R}^{n},
$$




$$
|G(x)| \leq m_{1}|x|^{p-1} \quad \text { for all } x \in \mathbb{R}^{n}
$$

and

$$
|\nabla F(x)| \leq m_{2}|x|^{p-1} \quad \text { for all } x \in \mathbb{R}^{n}
$$

$\left[H_{2}\right] e \in C\left(\mathbb{R}, \mathbb{R}^{n}\right)$ is a bounded function with $e(t) \neq \mathbf{0}=(0,0, \ldots, 0)^{T}$ and

$$
B:=\left(\int_{\mathbb{R}}|e(t)|^{q} d t\right)^{\frac{1}{q}}+\sup _{t \in \mathbb{R}}|e(t)|<+\infty .
$$

By (1.6) we know that $\left|e_{k}(t)\right| \leq \sup _{t \in \mathbb{R}}|e(t)|$. So for each $k \in \mathbb{N},\left(\int_{-k T}^{k T}\left|e_{k}(t)\right|^{q} d t\right)^{\frac{1}{q}}<B$ if $\left[H_{2}\right]$ holds. Let $C_{2 k T}=\left\{x \mid x \in C\left(\mathbb{R}, \mathbb{R}^{n}\right), x(t+2 k T) \equiv x(t)\right\}, C_{2 k T}^{1}=\left\{x \mid x \in C^{1}\left(\mathbb{R}, \mathbb{R}^{n}\right), x(t+\right.$ $2 k T) \equiv x(t)\}$, and $|x|_{0}=\max _{t \in[0,2 k T]}|x(t)|$. If the norms of $C_{2 k T}$ and $C_{2 k T}^{1}$ are respectively defined by $\|\cdot\|_{C_{2 k T}}=|\cdot|_{0}$ and $\|\cdot\|_{C_{2 k T}^{1}}=\max \left\{|x|_{0},\left|x^{\prime}\right|_{0}\right\}$, then $C_{2 k T}$ and $C_{2 k T}^{1}$ are Banach spaces. By $\langle\cdot, \cdot\rangle: \mathbb{R}^{n} \times \mathbb{R}^{n} \rightarrow \mathbb{R}$ we denote the standard inner product, and by $|\cdot|$ we denote the absolute value and the Euclidean norm on $\mathbb{R}^{n}$. For $\varphi \in C_{2 k T}$, set $\|\varphi\|_{r}=\left(\int_{-k T}^{k T}|\varphi(t)|^{r} d t\right)^{\frac{1}{r}}$, $r>1$. Let $\gamma \in C^{1}(\mathbb{R}, \mathbb{R})$ with $\gamma^{\prime}(t)<1$ for all $t \in[0, T]$. Let $\sigma_{0}=\min _{t \in[0, T]} \gamma^{\prime}(t)$ and $\sigma_{1}=$ $\max _{t \in[0, T]} \gamma^{\prime}(t)$. Define the linear operator

$$
A: C_{T} \rightarrow C_{T}, \quad[A x](t)=x(t)-C x(t-\tau) .
$$

\section{Main results}

First, we study some properties of all possible $2 k T$-periodic solutions of the following equation:

$$
\left(\varphi_{p}(u(t)-C u(t-\tau))^{\prime}\right)^{\prime}+\lambda \frac{d}{d t} \nabla F(u(t))+\lambda G(u(t-\gamma(t)))=\lambda e_{k}(t), \quad \lambda \in(0,1]
$$

Let $\Sigma \subset C_{2 k T}^{1}, k \in \mathbb{N}$, be the set of all the $2 k T$-periodic solutions to Eq. (3.1).

Theorem 3.1 If assumptions $\left[H_{1}\right]-\left[H_{2}\right]$ hold and

$$
\frac{\left(1-\sigma_{0}\right)^{p-1} \lambda_{M}^{\frac{p}{2}}\left[m_{1}\left(2|\gamma|_{0}+|\tau|\right)\left(1-\sigma_{1}\right)^{-\frac{1}{q}}+m_{2}\right]^{p}}{m_{0}^{p-1}}<1,
$$

where $\lambda_{M}=\max \left\{c_{i}^{2}\right\},\left|c_{i}\right| \neq 1, i=1,2, \ldots, n$, and $u \in \Sigma$ for each $k \in \mathbb{N}$, then

$$
\|u\|_{p} \leq A_{0}, \quad\left\|u^{\prime}\right\|_{p} \leq A_{1}, \quad|u|_{0} \leq \rho_{0}, \quad\left|u^{\prime}\right|_{0} \leq \rho_{1}
$$

where $A_{0}, A_{1}, \rho_{0}$, and $\rho_{1}$ are positive constants independent of $\lambda$ and $k$.

Proof If $u \in \Sigma$ and $k \in \mathbb{N}$, then $u$ satisfies

$$
\left(\varphi_{p}(u(t)-C u(t-\tau))^{\prime}\right)^{\prime}+\lambda \frac{d}{d t} \nabla F(u(t))+\lambda G(u(t-\gamma(t)))=\lambda e_{k}(t), \quad \lambda \in(0,1]
$$


Multiplying both sides of Eq. (3.2) by $[A u](t)$ and integrating from $-k T$ to $k T$, we get

$$
\begin{aligned}
& -\left\|A u^{\prime}\right\|_{p}^{p}+\lambda \int_{-k T}^{k T}\left\langle[A u](t), \frac{d}{d t} \nabla F(u(t))\right\rangle d t+\lambda \int_{-k T}^{k T}\langle[A u](t), G(u(t-\gamma(t)))\rangle d t \\
& =\lambda \int_{-k T}^{k T}\left\langle[A u](t), e_{k}(t)\right\rangle d t .
\end{aligned}
$$

Since

$$
\int_{-k T}^{k T}\left\langle[A u](t), \frac{d}{d t} \nabla F(u(t))\right\rangle d t=\int_{-k T}^{k T}\left\langle C u^{\prime}(t-\tau), \nabla F(u(t))\right\rangle d t,
$$

we have

$$
\begin{aligned}
& \lambda \int_{-k T}^{k T}\left\langle[A u](t), e_{k}(t)\right\rangle d t \\
& =-\left\|A u^{\prime}\right\|_{p}^{p}+\lambda \int_{-k T}^{k T}\left\langle C u^{\prime}(t-\tau), \nabla F(u(t))\right\rangle d t \\
& \quad+\lambda \int_{-k T}^{k T}\langle u(t)-u(t-\gamma(t)), G(u(t-\gamma(t)))\rangle d t \\
& \quad+\lambda \int_{-k T}^{k T}\langle(E-C) u(t-\gamma(t)), G(u(t-\gamma(t)))\rangle d t \\
& \quad-\lambda \int_{-k T}^{k T}\langle C u(t-\tau)-C u(t-\gamma(t)), G(u(t-\gamma(t)))\rangle d t
\end{aligned}
$$

and by assumption $\left[H_{1}\right]$

$$
\begin{aligned}
\left\|A u^{\prime}\right\|_{p}^{p}+\lambda m_{0} \int_{-k T}^{k T}|u(t-\gamma(t))|^{p} d t \\
\leq \lambda m_{1} \int_{-k T}^{k T}|u(t)-u(t-\gamma(t))||u(t-\gamma(t))|^{p-1} d t \\
\quad+\lambda m_{1} \lambda_{M}^{\frac{1}{2}} \int_{-k T}^{k T}|u(t-\tau)-u(t-\gamma(t))||u(t-\gamma(t))|^{p-1} d t \\
+\left|\lambda \int_{-k T}^{k T}\left\langle[A u](t), e_{k}(t)\right\rangle d t\right|+\left|\lambda \int_{-k T}^{k T}\left\langle C u^{\prime}(t-\tau), \nabla F(u(t))\right\rangle d t\right|
\end{aligned}
$$

where $\lambda_{M}=\max \left\{c_{i}^{2}\right\}, i=1,2, \ldots, n$.

By applying Lemma 2.2, Lemma 2.4, $\left[H_{1}\right]$, and $\left[H_{2}\right]$ we get

$$
\begin{aligned}
\frac{1}{1-\sigma_{0}}\|u\|_{p}^{p} & \leq \int_{-k T}^{k T}|u(t-\gamma(t))|^{p} d t=\int_{-k T}^{k T} \frac{1}{1-\gamma^{\prime}(\mu(t))}|u(t)|^{p} d t \\
& \leq \frac{1}{1-\sigma_{1}}\|u\|_{p}^{p}
\end{aligned}
$$

and

$$
\int_{-k T}^{k T}|u(t)-u(t-\gamma(t))||u(t-\gamma(t))|^{p-1} d t
$$




$$
\begin{aligned}
& \leq\left(\int_{-k T}^{k T}|u(t)-u(t-\gamma(t))|^{p} d t\right)^{p}\left(\int_{-k T}^{k T}|u(t-\gamma(t))|^{p} d t\right)^{\frac{p-1}{p}} \\
& \leq|\gamma|_{0} \frac{1}{\left(1-\sigma_{1}\right)^{\frac{p-1}{p}}}\left\|u^{\prime}\right\|_{p}\|u\|_{p}^{p-1} .
\end{aligned}
$$

Using the same method as for (3.5), we have

$$
\begin{aligned}
& \int_{-k T}^{k T}|u(t-\tau)-u(t-\gamma(t)) \| u(t-\gamma(t))|^{p-1} d t \\
& \leq\left(|\gamma|_{0}+|\tau|\right) \frac{1}{\left(1-\sigma_{1}\right)^{\frac{p-1}{p}}}\left\|u^{\prime}\right\|_{p}\|u\|_{p}^{p-1}
\end{aligned}
$$

and

$$
\begin{aligned}
& \left|\int_{-k T}^{k T}\left\langle[A u](t), e_{k}(t)\right\rangle d t\right| \\
& \quad \leq\left\|e_{k}\right\|_{q}\|u\|_{p}+\left\|e_{k}\right\|_{q}\|u\|_{p} \\
& \quad \leq B\left(1+\lambda_{M}^{\frac{1}{2}}\right)\|u\|_{p} .
\end{aligned}
$$

Furthermore, by $\left[H_{1}\right]$ we have

$$
\begin{aligned}
& \left|\int_{-k T}^{k T}\left\langle C u^{\prime}(t-\tau), \nabla F(u(t))\right\rangle d t\right| \\
& \quad \leq\left(\int_{-k T}^{k T}\left|C u^{\prime}(t-\tau)\right|^{p} d t\right)^{\frac{1}{p}}\left(\int_{-k T}^{k T}|\nabla F(u(t))|^{q} d t\right)^{\frac{1}{q}} \\
& \quad \leq \lambda_{M}^{\frac{1}{2}} m_{2}\left\|u^{\prime}\right\|_{p}\|u\|_{p}^{p-1} .
\end{aligned}
$$

Applying (3.4)-(3.8) to (3.3), we obtain

$$
\begin{aligned}
\left\|A u^{\prime}\right\|_{p}^{p} & +\lambda m_{0} \frac{1}{1-\sigma_{0}}\|u\|_{p}^{p} \\
\leq & \lambda \lambda_{M}^{\frac{1}{2}}\left[m_{1}\left(2|\gamma|_{0}+|\tau|\right)\left(1-\sigma_{1}\right)^{-\frac{1}{q}}\right. \\
& \left.+\lambda m_{2}\right]\left\|u^{\prime}\right\|_{p}\|u\|_{p}^{p-1}+\lambda B\left(1+\lambda_{M}^{\frac{1}{2}}\right)\|u\|_{p} .
\end{aligned}
$$

By (3.9) we get

$$
\begin{aligned}
\|u\|_{p}^{p} \leq & \frac{1-\sigma_{0}}{m_{0}} \lambda_{M}^{\frac{1}{2}}\left[m_{1}\left(2|\gamma|_{0}+|\tau|\right)\left(1-\sigma_{1}\right)^{-\frac{1}{q}}+m_{2}\right]\left\|u^{\prime}\right\|_{p}\|u\|_{p}^{p-1} \\
& +\frac{1-\sigma_{0}}{m_{0}} B\left(1+\lambda_{M}^{\frac{1}{2}}\right)\|u\|_{p} .
\end{aligned}
$$

Since

$$
\frac{\left(1-\sigma_{0}\right)^{p-1} \lambda_{M}^{\frac{p}{2}}\left[m_{1}\left(2|\gamma|_{0}+|\tau|\right)\left(1-\sigma_{1}\right)^{-\frac{1}{q}}+m_{2}\right]^{p}}{m_{0}^{p-1}}<1,
$$


Gao and Chen Advances in Difference Equations

(2018) 2018:446

Page 7 of 15

there exists a constant $\varepsilon_{0} \in(0,1)$ such that

$$
\frac{\left(1-\sigma_{0}\right)^{p-1} \lambda_{M}^{\frac{p}{2}}\left[m_{1}\left(2|\gamma|_{0}+|\tau|\right)\left(1-\sigma_{1}\right)^{-\frac{1}{q}}+m_{2}\right]^{p}}{\left(1-\varepsilon_{0}\right)^{p-1} m_{0}^{p-1}}<1 .
$$

Applying Lemma 2.3 and (3.10), we get

$$
\begin{aligned}
\|u\|_{p}^{p} & \\
\leq & \max \left\{\frac{\left(1-\sigma_{0}\right)^{p} \lambda_{M}^{\frac{p}{2}}\left[m_{1}\left(2|\gamma|_{0}+|\tau|\right)\left(1-\sigma_{1}\right)^{-\frac{1}{q}}+m_{2}\right]^{p}}{\left(1-\varepsilon_{0}\right)^{p} m_{0}^{p}}\left\|u^{\prime}\right\|_{p}^{p},\right. \\
& {\left.\left[\frac{1-\sigma_{0}}{\varepsilon_{0} m_{0}} B\left(1+\lambda_{M}^{\frac{1}{2}}\right)\right]^{\frac{p}{p-1}}\right\} . }
\end{aligned}
$$

If

$$
\frac{\left(1-\sigma_{0}\right)^{p} \lambda_{M}^{\frac{p}{2}}\left[m_{1}\left(2|\gamma|_{0}+|\tau|\right)\left(1-\sigma_{1}\right)^{-\frac{1}{q}}+m_{2}\right]^{p}}{\left(1-\varepsilon_{0}\right)^{p} m_{0}^{p}}\left\|u^{\prime}\right\|_{p}^{p} \leq\left[\frac{1-\sigma_{0}}{\varepsilon_{0} m_{0}} B\left(1+\lambda_{M}^{\frac{1}{2}}\right)\right]^{\frac{p}{p-1}}
$$

then

$$
\begin{aligned}
\|u\|_{p}^{p} & \leq\left[\frac{1-\sigma_{0}}{\varepsilon_{0} m_{0}} B\left(1+\lambda_{M}^{\frac{1}{2}}\right)\right]^{\frac{p}{p-1}}, \quad\|u\|_{p}^{p-1} \leq \frac{1-\sigma_{0}}{\varepsilon_{0} m_{0}} B\left(1+\lambda_{M}^{\frac{1}{2}}\right) \\
\|u\|_{p} & \leq\left[\frac{1-\sigma_{0}}{\varepsilon_{0} m_{0}} B\left(1+\lambda_{M}^{\frac{1}{2}}\right)\right]^{\frac{1}{p-1}} .
\end{aligned}
$$

By Lemma 2.6 we have $\left\|u^{\prime}\right\|_{p}=\left\|A^{-1} A u^{\prime}\right\|_{p} \leq \alpha^{\frac{1}{p}}\left\|A u^{\prime}\right\|_{p}$. From (3.9) and Lemma 2.3 with $\varepsilon=\frac{1}{2}$ we get

$$
\begin{aligned}
& \left\|A u^{\prime}\right\|_{p}^{p} \\
& \leq \alpha^{\frac{1}{p}} \lambda_{M}^{\frac{1}{2}}\left[m_{1}\left(2|\gamma|_{0}+|\tau|\right)\left(1-\sigma_{1}\right)^{-\frac{1}{q}}+m_{2}\right] \frac{1-\sigma_{0}}{\varepsilon_{0} m_{0}} B\left(1+\lambda_{M}^{\frac{1}{2}}\right)\left\|A u^{\prime}\right\|_{p} \\
& \quad+\left(\frac{1-\sigma_{0}}{\varepsilon_{0} m_{0}}\right)^{\frac{1}{p-1}} B\left(1+\lambda_{M}^{\frac{1}{2}}\right)^{\frac{p}{p-1}}
\end{aligned}
$$

and

$$
\begin{aligned}
& \left\|A u^{\prime}\right\|_{p} \\
& \leq \max \left\{2^{\frac{1}{p-1}}\left[\alpha^{\frac{1}{p}} \lambda_{M}^{\frac{1}{2}}\left[m_{1}\left(2|\gamma|_{0}+|\tau|\right)\left(1-\sigma_{1}\right)^{-\frac{1}{q}}+m_{2}\right] \frac{1-\sigma_{0}}{\varepsilon_{0} m_{0}} B\left(1+\lambda_{M}^{\frac{1}{2}}\right)\right]^{\frac{1}{p-1}},\right. \\
& \left.\quad 2^{\frac{1}{p}}\left(\frac{1-\sigma_{0}}{\varepsilon_{0} m_{0}}\right)^{\frac{1}{p(p-1)}} B\left(1+\lambda_{M}^{\frac{1}{2}}\right)^{\frac{1}{p-1}}\right\}:=M_{1} .
\end{aligned}
$$

If

$$
\frac{\left(1-\sigma_{0}\right)^{p} \lambda_{M}^{\frac{p}{2}}\left[m_{1}\left(2|\gamma|_{0}+|\tau|\right)\left(1-\sigma_{1}\right)^{-\frac{1}{q}}+m_{2}\right]^{p}}{\left(1-\varepsilon_{0}\right)^{p} m_{0}^{p}}\left\|u^{\prime}\right\|_{p}^{p} \geq\left[\frac{1-\sigma_{0}}{\varepsilon_{0} m_{0}} B\left(1+\lambda_{M}^{\frac{1}{2}}\right)\right]^{\frac{p}{p-1}}
$$


then

$$
\begin{aligned}
& \|u\|_{p}^{p} \leq \frac{\left(1-\sigma_{0}\right)^{p^{p}} \lambda_{M}^{\frac{p}{2}}\left[m_{1}\left(2|\gamma|_{0}+|\tau|\right)\left(1-\sigma_{1}\right)^{-\frac{1}{q}}+m_{2}\right]^{p}}{\left(1-\varepsilon_{0}\right)^{p} m_{0}^{p}}\left\|u^{\prime}\right\|_{p}^{p} \\
& \|u\|_{p}^{p-1} \leq\left[\frac{\left(1-\sigma_{0}\right)^{p} \lambda_{M}^{\frac{p}{2}}\left[m_{1}\left(2|\gamma|_{0}+|\tau|\right)\left(1-\sigma_{1}\right)^{-\frac{1}{q}}+m_{2}\right]^{p}}{\left(1-\varepsilon_{0}\right)^{p} m_{0}^{p}}\right]^{\frac{p-1}{p}}\left\|u^{\prime}\right\|_{p}^{p-1},
\end{aligned}
$$

and

$$
\|u\|_{p} \leq\left[\frac{\left(1-\sigma_{0}\right)^{p} \lambda_{M}^{\frac{p}{2}}\left[m_{1}\left(2|\gamma|_{0}+|\tau|\right)\left(1-\sigma_{1}\right)^{-\frac{1}{q}}+m_{2}\right]^{p}}{\left(1-\varepsilon_{0}\right)^{p} m_{0}^{p}}\right]^{\frac{1}{p}}\left\|u^{\prime}\right\|_{p} .
$$

From (3.9) we have

$$
\begin{aligned}
& \left\|A u^{\prime}\right\|_{p}^{p} \\
& \leq \leq \frac{\left(1-\sigma_{0}\right)^{p-1} \lambda_{M}^{\frac{p}{2}}\left[m_{1}\left(2|\gamma|_{0}+|\tau|\right)\left(1-\sigma_{1}\right)^{-\frac{1}{q}}+m_{2}\right]^{p}}{\left(1-\varepsilon_{0}\right)^{p-1} m_{0}^{p-1}}\left\|A u^{\prime}\right\|_{p}^{p} \\
& \quad+\alpha^{\frac{1}{p}} B\left(1+\lambda_{M}^{\frac{1}{2}}\right) \frac{\left(1-\sigma_{0}\right) \lambda_{M}^{\frac{1}{2}}\left[m_{1}\left(2|\gamma|_{0}+|\tau|\right)\left(1-\sigma_{1}\right)^{-\frac{1}{q}}+m_{2}\right]}{\left(1-\varepsilon_{0}\right) m_{0}^{p}}\left\|A u^{\prime}\right\|_{p} .
\end{aligned}
$$

Combining this with (3.11), we see that there exists a constant $M_{2}>0$ such that

$$
\left\|A u^{\prime}\right\|_{p} \leq M_{2}
$$

Obviously,

$$
\begin{aligned}
& \left\|A u^{\prime}\right\|_{p} \leq \max \left\{M_{1}, M_{2}\right\}:=M, \\
& \left\|u^{\prime}\right\|_{p} \leq \alpha^{\frac{1}{p}}\left\|A u^{\prime}\right\|_{p} \leq \alpha^{\frac{1}{p}} M:=A_{1}, \\
& \|u\|_{p} \\
& \leq \max \left\{\left[\frac{1-\sigma_{0}}{\varepsilon_{0} m_{0}} B\left(1+\lambda_{M}^{\frac{1}{2}}\right)\right]^{\frac{1}{p-1}},\right. \\
& \left.\quad\left[\frac{\left(1-\sigma_{0}\right)^{p} \lambda_{M}^{\frac{p}{2}}\left[m_{1}\left(2|\gamma|_{0}+|\tau|\right)\left(1-\sigma_{1}\right)^{-\frac{1}{q}}+m_{2}\right]^{p}}{\left(1-\varepsilon_{0}\right)^{p} m_{0}^{p}}\right]^{\frac{1}{p}} A_{1}\right\}:=A_{0} .
\end{aligned}
$$

By (3.15) we can easily notice that $A_{0}$ and $A_{1}$ are constants independent of $\lambda$ and $k$. By Lemma 2.1, for $t \in[-k T, k T]$, we obtain

$$
\begin{aligned}
|u(t)| & \leq(2 T)^{-\frac{1}{p}}\left(\int_{t-T}^{t+T}|u(s)|^{p} d s\right)^{\frac{1}{p}}+T(2 T)^{-\frac{1}{p}}\left(\int_{t-T}^{t+T}\left|u^{\prime}(s)\right|^{p} d s\right)^{\frac{1}{p}} \\
& \leq(2 T)^{-\frac{1}{p}}\left(\int_{t-k T}^{t+k T}|u(s)|^{p} d s\right)^{\frac{1}{p}}+T(2 T)^{-\frac{1}{p}}\left(\int_{t-k T}^{t+k T}\left|u^{\prime}(s)\right|^{p} d s\right)^{\frac{1}{p}} \\
& =(2 T)^{-\frac{1}{p}}\left(\int_{-k T}^{k T}|u(s)|^{p} d s\right)^{\frac{1}{p}}+T(2 T)^{-\frac{1}{p}}\left(\int_{-k T}^{k T}\left|u^{\prime}(s)\right|^{p} d s\right)^{\frac{1}{p}} .
\end{aligned}
$$


From (3.13) and (3.14) we have

$$
\begin{aligned}
|u|_{0} & \leq(2 T)^{-\frac{1}{p}}\|u\|_{p}+T(2 T)^{-\frac{1}{p}}\left\|u^{\prime}\right\|_{p} \\
& \leq(2 T)^{-\frac{1}{p}} A_{0}+T(2 T)^{-\frac{1}{p}} A_{1}:=\rho_{0} .
\end{aligned}
$$

Furthermore, setting $F_{\rho_{0}}:=\max _{|x| \leq \rho_{0}}|\nabla F(x)|$ and $G_{\rho_{0}}:=\max _{|x| \leq \rho_{0}}|G(x)|$, by Eq. (3.2) we get

$$
\left|\frac{d}{d t}\left[\varphi_{p}\left(\left[A u^{\prime}\right](t)\right)+\lambda \nabla F(u(t))\right]\right| \leq G_{\rho_{0}}+\sup _{t \in \mathbb{R}}|e(t)|:=\tilde{\rho}, \quad t \in[-k T, k T] .
$$

Combining the continuity of $\left[A u^{\prime}\right](t)$ and (3.13), we find that there exists $t_{i} \in[i T,(i+1) T]$, $i=-k,-k+1, \ldots, k-1$, such that

$$
\begin{aligned}
\left|\left[A u^{\prime}\right]\left(t_{i}\right)\right| & =\left|\frac{1}{T} \int_{i T}^{(i+1) T}\left[A u^{\prime}\right](s) d s\right| \\
& \leq \frac{1}{T} \int_{i T}^{(i+1) T}\left|\left[A u^{\prime}\right](s)\right| d s \\
& \leq T^{\frac{1-q}{q}}\left(\int_{i T}^{(i+1) T}\left|\left[A u^{\prime}\right](s)\right|^{p} d s\right)^{\frac{1}{p}} \\
& \leq T^{\frac{1-q}{q}}\left(\int_{-k T}^{k T}\left|\left[A u^{\prime}\right](s)\right|^{p} d s\right)^{\frac{1}{p}} \\
& \leq T^{\frac{1-q}{q}} \max \left\{M_{1}, M_{2}\right\} .
\end{aligned}
$$

By (3.16)-(3.18) we have

$$
\begin{aligned}
& \left|\varphi_{p}\left(\left[A u^{\prime}\right](t)\right)+\lambda \nabla F(u(t))\right| \\
& \quad \leq\left|\int_{t_{i}}^{t} \frac{d}{d s}\left[\varphi_{p}\left(\left[A u^{\prime}\right](s)\right)+\lambda \nabla F(u(s))\right] d s+\varphi_{p}\left(\left[A u^{\prime}\right]\left(t_{i}\right)\right)+\lambda \nabla F\left(u\left(t_{i}\right)\right)\right| \\
& \quad \leq \int_{i T}^{(i+1) T}\left|\left[\varphi_{p}\left(\left[A u^{\prime}\right](s)\right)+\lambda \nabla F(u(s))\right]\right| d s+\left|\varphi_{p}\left(\left[A u^{\prime}\right]\left(t_{i}\right)\right)\right|+F_{\rho_{0}} \\
& \quad \leq \tilde{\rho} T+\left[T^{\frac{1-q}{q}} \max \left\{M_{1}, M_{2}\right\}\right]^{p-1}+F_{\rho_{0}}:=\rho,
\end{aligned}
$$

which yields

$$
\left|\left[A u^{\prime}\right](t)\right| \leq\left[\rho+F_{\rho_{0}}\right]^{\frac{1}{p-1}}
$$

It follows from Lemma 2.6 and (3.19) that

$$
\left|u^{\prime}\right|_{0}=\left\|A^{-1} A u^{\prime}\right\| \leq\left(\sum_{i=1}^{n} \frac{1}{|1-| c_{i}||}\right)\left\|A u^{\prime}\right\| \leq\left(\sum_{i=1}^{n} \frac{1}{|1-| c_{i}||}\right)\left[\rho+F_{\rho_{0}}\right]^{\frac{1}{p-1}}:=\rho_{1} .
$$

Note that $\rho_{1}$ is independent of $\lambda$ and $k$. The proof of Theorem 3.1 is completed. 
Theorem 3.2 If the conditions of Theorem 3.1 are satisfied, then Eq. (3.2) has at least one $2 k T$-periodic solution $u_{k}(t)$ for each $k \in \mathbb{N}$ such that

$$
\left\|u_{k}\right\|_{p} \leq A_{0}, \quad\left\|u_{k}^{\prime}\right\|_{p} \leq A_{1}, \quad\left|u_{k}\right|_{0} \leq \rho_{0}, \quad\left|u_{k}^{\prime}\right|_{0} \leq \rho_{1}
$$

Proof To apply Lemma 2.5, we study the $p$-Laplacian neutral systems

$$
\left(\varphi_{p}(u(t)-C u(t-\tau))^{\prime}\right)^{\prime}+\lambda \frac{d}{d t} \nabla F(u(t))+\lambda G(u(t-\gamma(t)))=\lambda e_{k}(t), \quad \lambda \in(0,1) .
$$

Let $\Omega_{1} \subset C_{2 k T}^{1}$ be the set of all $2 k T$-periodic of Eq. (3.20). From Theorem 3.1, assuming that $u \in \Omega_{1} \subset \Sigma$ by $(0,1) \subset(0,1]$, we get

$$
|u|_{0} \leq \rho_{0}, \quad\left|u^{\prime}\right|_{0} \leq \rho_{1} .
$$

Set $\Omega_{2}=\{x: x \in \operatorname{Ker} L, Q N x=0\}$,

$$
\begin{aligned}
& L: D(L) \subset C_{2 k T} \rightarrow C_{2 k T}, \quad L u=\left(\varphi_{p}(A u)^{\prime}\right)^{\prime}, \\
& N: C_{2 k T} \rightarrow C_{2 k T}^{1}, \quad N u=-\frac{d}{d t} \nabla F(u(t))-G(u(t-\gamma(t)))+e_{k}(t), \\
& Q: C_{2 k T} \rightarrow C_{2 k T} / \operatorname{Im} L, \quad Q y=\frac{1}{2 k T} \int_{-k T}^{k T} y(s) d s .
\end{aligned}
$$

Obviously, $x=a \in \mathbb{R}^{n}$ when $x \in \Omega_{2}$. Meanwhile, it follows from $\left[H_{1}\right]$ that

$$
2 k T m_{0}|a|^{p} \leq \int_{-k T}^{k T}\left|\left\langle(E-C) a, e_{k}(t)\right\rangle\right| d t \leq B|a|\left(1+\left|c_{M}\right|\right)(2 k T)^{\frac{1}{p}},
$$

that is,

$$
|a| \leq m_{0}^{\frac{1}{1-p}} B^{\frac{1}{p-1}} T^{\frac{-1}{p}}\left(1+\left|c_{M}\right|\right)^{\frac{1}{p-1}}:=B_{0},
$$

where $\left|c_{M}\right|=\max \left|c_{i}\right|, i=1,2, \ldots, n$.

Let $\Omega=\left\{x: x \in C_{2 k T}^{1},|x|_{0}<\rho_{0}+B_{0},\left|x^{\prime}\right|_{0}<\rho_{1}+1\right\}$. Then $\Omega \supset \Omega_{1} \cup \Omega_{2}$. Thus assumptions $\left[A_{1}\right]$ and $\left[A_{2}\right]$ of Lemma 2.5 are satisfied. Next, we can prove that $\left[A_{3}\right]$ of Lemma 2.5 is also satisfied. Let

$$
H(x, \mu):\left(\Omega \cap \mathbb{R}^{n}\right) \times[0,1] \longrightarrow \mathbb{R}^{n}: H(x, \mu)=-\mu x+(1-\mu) \Delta(x),
$$

where $\Delta(x)=\frac{1}{2 k T} \int_{-k T}^{k T}\left[G(x)-e_{k}(t)\right] d t$ is determined by Lemma 2.5. By $\left[H_{1}\right]$ we get

$$
H(x, \mu) \neq 0, \quad \forall(x, \mu) \in\left[\partial\left(\Omega \cap \mathbb{R}^{n}\right)\right] \times[0,1] .
$$

Thus

$$
\begin{aligned}
& \operatorname{deg}\{J Q N, \Omega \cap \operatorname{Ker} L, 0\} \\
& \quad=\operatorname{deg}\{H(x, 0), \Omega \cap \operatorname{Ker} L, 0\}
\end{aligned}
$$


$=\operatorname{deg}\{H(x, 1), \Omega \cap \operatorname{Ker} L, 0\}$

$\neq 0$.

So, $A_{3}$ of Lemma 2.5 holds. By Lemma $2.5, u_{k} \in \bar{\Omega}$ is a $2 k T$-periodic solution for Eq. (1.2) when $\lambda=1$. Therefore, by means of Theorem 3.1 we have

$$
\left\|u_{k}\right\|_{p} \leq A_{0}, \quad\left\|u_{k}^{\prime}\right\|_{p} \leq A_{1}, \quad\left|u_{k}\right|_{0} \leq \rho_{0}, \quad\left|u_{k}^{\prime}\right|_{0} \leq \rho_{1}
$$

Theorem 3.3 Assume that the conditions in Theorem 3.1 are satisfied. Then Eq. (1.1) has a nontrivial homoclinic solution.

Proof By Theorem 3.2, Eq. (1.5) has a $2 k T$-periodic solution $u_{k}(t)$ for each $k \in \mathbb{N}$. Thus $u_{k}(t)$ satisfies

$$
\left(\varphi_{p}\left(u_{k}(t)-C u_{k}(t-\tau)\right)^{\prime}\right)^{\prime}=-\frac{d}{d t} \nabla F\left(u_{k}(t)\right)-G\left(u_{k}(t-\gamma(t))\right)+e_{k}(t) .
$$

Set $y_{k}=\varphi_{p}\left(A u_{k}^{\prime}\right)$ for $k>k_{0}$. From (3.19) and (3.22) we see that

$$
\left|y_{k}\right|_{0} \leq \rho+F_{\rho_{0}}
$$

and

$$
\left|y_{k}^{\prime}\right|_{0} \leq \max _{|x| \leq \rho_{0}}\left(\sum_{i=1}^{n} \sum_{j=1}^{n}\left|\frac{\partial^{2} F(x)}{\partial x_{i} \partial x_{j}}\right|^{2}\right)^{\frac{1}{2}}\left|u_{k}^{\prime}\right|_{0}+G_{\rho_{0}}+\sup _{t \in R}|e(t)|:=\rho_{2} .
$$

By the method similar to that of Lemma 2.4 in [12] we can get that there is $u_{0} \in C^{1}\left(\mathbb{R}, \mathbb{R}^{n}\right)$ such that $u_{k_{j}}^{\prime}(t) \rightarrow u_{0}^{\prime}(t)$ uniformly on $[c, d] \subset \mathbb{R}$, where $\left\{u_{k_{j}}\right\}$ is a subsequence of $\left\{u_{k}\right\}$.

There exists $j_{0}>0$ such that $\left[a-|\gamma|_{0}, b+|\gamma|_{0}\right] \subset\left[-k_{j} T, k_{j} T-\varepsilon_{0}\right]$ with $j>j_{0}$ and $a<b \in \mathbb{R}$. Therefore, by (1.5) and (3.15), for $t \in\left[a-|\gamma|_{0}, b+|\gamma|_{0}\right]$, we get

$$
\left(\varphi_{p}\left(u_{k_{j}}(t)-C u_{k_{j}}(t-\tau)\right)^{\prime}\right)^{\prime}=-\frac{d}{d t} \nabla F\left(u_{k_{j}}(t)\right)-G\left(u_{k_{j}}(t-\gamma(t))\right)+e(t) .
$$

From (3.23) we get

$$
\begin{aligned}
y_{k}^{\prime} & =\left(\varphi_{p}\left(A u_{k_{j}}^{\prime}\right)\right)^{\prime} \\
& =-\frac{d}{d t} \nabla F\left(u_{k_{j}}(t)\right)-G\left(u_{k_{j}}(t-\gamma(t))\right)+e(t) \\
& \rightarrow-\frac{d}{d t} \nabla F\left(u_{0}(t)\right)-G\left(u_{0}(t-\gamma(t))\right)+e(t) \\
& :=\chi(t), \quad \text { uniformly on }[a, b],
\end{aligned}
$$

because $y_{k_{j}}^{\prime}(t)$ is continuously differentiable on $(a, b)$ for $j>j_{0}$ and $y_{k_{j}}^{\prime}(t) \rightarrow \chi(t)$ uniformly on $[a, b]$. We know that $\chi(t)=\left(\varphi_{p}\left(u_{0}(t)-C u_{0}(t-\tau)\right)^{\prime}\right)^{\prime}, t \in \mathbb{R}$. Since $a, b \in \mathbb{R}$ are arbitrary, $u_{0}(t)$ is a solution of $(1.1)$. 
Next, we prove that $u_{0}(t) \rightarrow 0$ and $u_{0}^{\prime}(t) \rightarrow 0$ as $|t| \rightarrow+\infty$. Since

$$
\begin{aligned}
\int_{-\infty}^{+\infty}\left(\left|u_{0}(t)\right|^{p}+\left|u_{0}^{\prime}(t)\right|^{p}\right) d t & =\lim _{i \rightarrow+\infty} \int_{-i T}^{i T}\left(\left|u_{0}(t)\right|^{p}+\left|u_{0}^{\prime}(t)\right|^{p}\right) d t \\
& =\lim _{i \rightarrow+\infty} \lim _{j \rightarrow+\infty} \int_{-i T}^{i T}\left(\left|u_{k_{j}}(t)\right|^{p}+\left|u_{k_{j}}^{\prime}(t)\right|^{p}\right) d t
\end{aligned}
$$

if $k_{j}>i, i \in \mathbb{N}$, then it follows from (3.14) and (3.15) that

$$
\int_{-i T}^{i T}\left(\left|u_{k_{j}}(t)\right|^{p}+\left|u_{k_{j}}^{\prime}(t)\right|^{p}\right) d t \leq \int_{-k_{j} T}^{k_{j} T}\left(\left|u_{k_{j}}(t)\right|^{p}+\left|u_{k_{j}}^{\prime}(t)\right|^{p}\right) d t \leq A_{0}^{p}+A_{1}^{p}
$$

Letting $i \rightarrow+\infty$ and $j \rightarrow+\infty$, we have

$$
\int_{-\infty}^{+\infty}\left(\left|u_{0}(t)\right|^{p}+\left|u_{0}^{\prime}(t)\right|^{p}\right) d t \leq A_{0}^{p}+A_{1}^{p}
$$

and

$$
\int_{|t| \geq r}\left(\left|u_{0}(t)\right|^{p}+\left|u_{0}^{\prime}(t)\right|^{p}\right) d t \rightarrow 0, \quad r \rightarrow+\infty
$$

From (3.13), similarly to the previous method, we get

$$
\int_{-\infty}^{+\infty}\left|u_{0}^{\prime}(t)-C u_{0}^{\prime}(t-\tau)\right|^{p} d t \leq M^{p}
$$

From Lemma 2.1 we can see that

$$
\begin{aligned}
\left|u_{0}(t)\right| & \leq(2 T)^{-\frac{1}{p}}\left(\int_{t-T}^{t+T}\left|u_{0}(s)\right|^{p} d s\right)^{\frac{1}{p}}+T(2 T)^{-\frac{1}{p}}\left(\int_{t-T}^{t+T}\left|u_{0}^{\prime}(s)\right|^{p} d s\right)^{\frac{1}{p}} \\
& \leq \max \left\{(2 T)^{-\frac{1}{p}}, T(2 T)^{-\frac{1}{p}}\right\} \int_{t-T}^{t+T}\left(\left|u_{0}(t)\right|^{p}+\left|u_{0}^{\prime}(t)\right|^{p}\right) d t \rightarrow 0, \quad|t| \rightarrow+\infty .
\end{aligned}
$$

Finally, we will prove that $\left|u_{0}^{\prime}(t)\right| \rightarrow 0$ as $|t| \rightarrow+\infty$ if the following condition holds:

$$
\left|\left[\tilde{A} u_{0}^{\prime}\right](t)\right|:=\left|u_{0}^{\prime}(t)-C u_{0}^{\prime}(t-\tau)\right| \rightarrow 0, \quad|t| \rightarrow+\infty
$$

On the one hand, from (3.16) we have $\left|u_{0}\right| \leq \rho_{0}$, and applying (1.1) yields

$$
\begin{aligned}
& \left|\frac{d}{d t}\left(\left|\left[\tilde{A} u_{0}^{\prime}\right](t)\right|^{p-2}\left[\tilde{A} u_{0}^{\prime}\right](t)\right)\right| \\
& \quad \leq\left|\frac{d}{d t} \nabla F\left(u_{0}(t)\right)\right|+\left|G\left(u_{0}(t-\gamma(t))\right)\right|+\sup _{t \in \mathbb{R}}|e(t)| \\
& \quad \leq \sup _{|u| \leq \rho_{0}}\left|\frac{d}{d t} \nabla F(u)\right|+\sup _{|u| \leq \rho_{0}}|G(u)|+\sup _{t \in \mathbb{R}}|e(t)|:=\tilde{M} \quad \text { for } t \in \mathbb{R} .
\end{aligned}
$$

If (3.27) does not hold, then there exist a parameter $\varepsilon_{0} \in\left(0, \frac{1}{2}\right)$ and a sequence $\left\{t_{k}\right\}$ such that

$$
\left|t_{1}\right|<\left|t_{2}\right|<\left|t_{3}\right|<\cdots, \quad\left|t_{k}\right|+1<\left|t_{k+1}\right|, \quad k=1,2, \ldots,
$$


and

$$
\left|\tilde{A} u_{0}^{\prime}\left(t_{k}\right)\right| \geq\left(2 \varepsilon_{0}\right)^{\frac{1}{p-1}}, \quad k=1,2, \ldots
$$

So, for $t \in\left[t_{k}, t_{k}+\varepsilon_{0} /(1+\tilde{M})\right]$, we have

$$
\begin{aligned}
\left|\left[\tilde{A} u_{0}^{\prime}\right](t)\right|^{p-1} & =\left.||\left[\tilde{A} u_{0}^{\prime}\right]\left(t_{k}\right)\right|^{p-2}\left[\tilde{A} u_{0}^{\prime}\right]\left(t_{k}\right)+\int_{t_{k}}^{t} \frac{d}{d s}\left(\left|\left[\tilde{A} u_{0}^{\prime}\right](s)\right|^{p-2}\left[\tilde{A} u_{0}^{\prime}\right](s)\right) d s \mid \\
& \geq\left|\left[\tilde{A} u_{0}^{\prime}\right]\left(t_{k}\right)\right|^{p-1}-\int_{t_{k}}^{t}\left|\frac{d}{d s}\right|\left(\left.\left[\tilde{A} u_{0}^{\prime}\right](s)\right|^{p-2}\left[\tilde{A} u_{0}^{\prime}\right](s)\right) \mid d s \\
& \geq \varepsilon_{0} .
\end{aligned}
$$

Note that

$$
\int_{-\infty}^{+\infty}\left|\left[\tilde{A} u_{0}^{\prime}\right]\left(t_{k}\right)\right|^{p} d t \geq \sum_{k=1}^{\infty} \int_{t_{k}}^{t_{k}+\varepsilon_{0} /(1+\tilde{M})}\left|\left[\tilde{A} u_{0}^{\prime}\right]\left(t_{k}\right)\right|^{p} d t=\infty
$$

which contradicts (3.26), and thus (3.27) holds.

On the other hand, let $u_{0}^{\prime}(t)=\left(u_{0_{1}}^{\prime}(t), u_{0_{2}}^{\prime}(t), \ldots, u_{0_{n}}^{\prime}(t)\right)$. From (3.21) we know that $\left|A u_{k}^{\prime}\right|<$ $\left(1+\sqrt{\sum_{i=1}^{n}\left|c_{i}\right|^{2}}\right) \rho_{1}:=B_{1}$. For all $\varepsilon>0$, let $N=\left[\log _{\left|c_{i}\right|}^{\frac{\varepsilon\left(1-\left|c_{i}\right|\right)}{2 B_{1}}}\right]>0$. Then $\sum_{h=N+1}^{\infty}\left|c_{i}\right|^{h}<\frac{\varepsilon}{2 B_{1}}$ $\left(\left|c_{i}\right|<1\right)$. According to (3.27), it is easy to find that there exists a constant $G>0$ such that $\left|u_{0_{i}}^{\prime}(t)-c_{i} u_{0_{i}}^{\prime}(t-\tau)\right|<\frac{\varepsilon}{2(N+1)}$ for $t>G$. Set $P_{T}=\{x \mid x \in C(\mathbb{R}, \mathbb{R}), x(t+T) \equiv x(t)\}$ and $A_{0}: P_{T} \rightarrow P_{T},\left[A_{0} x\right](t)=x(t)-c x(t-\tau)$ with $|c| \neq 1$. Then applying Lemma 2.3 in [13], we obtain

$$
\left[A_{0}^{-1} f\right](t)= \begin{cases}\sum_{j \geq 0} c^{j} f(t-j \tau), & |c|<1 \forall f \in P_{T}, \\ -\sum_{j \geq 0} c^{-j} f(t+j \tau), & |c|>1 \forall f \in P_{T} .\end{cases}
$$

When $\left|c_{i}\right|<1$, this yields

$$
\begin{aligned}
& \left|u_{0_{i}}^{\prime}(t)\right| \\
& \quad=\lim _{j \rightarrow+\infty}\left|\left[A^{-1} A u_{k_{j_{i}}}^{\prime}\right](t)\right| \\
& \quad \leq\left|\lim _{j \rightarrow \infty} \sum_{h \geq 0}^{N} c_{i}^{h}\left[A u_{k_{j_{0_{i}}}}^{\prime}\right](t-h \tau)+\sum_{h=N+1}^{\infty} c_{i}^{h}\left[A u_{k_{j_{i}}}^{\prime}\right](t-h \tau)\right| \\
& \quad \leq\left|\lim _{j \rightarrow \infty} \sum_{h \geq 0}^{N} c_{i}^{h}\left[A u_{k_{j_{0_{i}}}}^{\prime}\right](t-h \tau)\right|+\left|\lim _{j \rightarrow \infty} \sum_{h=N+1}^{\infty} c_{i}^{h}\left[A u_{k_{j_{i}}}^{\prime}\right](t-h \tau)\right| \\
& \quad \leq \lim _{j \rightarrow \infty} \sum_{h \geq 0}^{N}\left|c_{i}\right|^{h}\left|\left[A u_{k_{j_{i}}}^{\prime}\right](t-h \tau)\right|+B_{1} \sum_{h=N+1}^{\infty}\left|c_{i}\right|^{h} \\
& \quad=\sum_{h \geq 0}^{N}\left|c_{i}\right|^{h}\left|\left(u_{0_{i}}^{\prime}(t-h \tau)-c_{i} u_{0_{i}}^{\prime}(t-(h+1) \tau)\right)\right|+B_{1} \sum_{h=N+1}^{\infty}\left|c_{i}\right|^{h} .
\end{aligned}
$$


By (3.28), for arbitrary $\varepsilon>0$, there exists $\bar{N}=G+N$ such that, for $t>\bar{N}$,

$$
\begin{aligned}
\left|u_{0_{i}}^{\prime}(t)\right| & \leq \sum_{h \geq 0}^{N}\left|c_{i}\right|^{h}\left|\left(u_{0_{i}}^{\prime}(t-h \tau)-c_{i} u_{0_{i}}^{\prime}(t-(h+1) \tau)\right)\right|+\left|B_{1} \sum_{h=N+1}^{\infty} c_{i}^{h}\right| \\
& <(N+1) \frac{\varepsilon}{2(N+1)}+B_{1} \frac{\varepsilon}{2 B_{1}} \\
& =\varepsilon .
\end{aligned}
$$

So, $\left|u_{0_{i}}^{\prime}(t)\right| \rightarrow 0$ as $|t| \rightarrow+\infty$. Similarly to the previous method, when $\left|c_{i}\right|>1,\left|u_{0_{i}}^{\prime}(t)\right| \rightarrow 0$ also holds as $|t| \rightarrow+\infty$. Thus $\left|u_{0}^{\prime}(t)\right| \rightarrow 0$ as $|t| \rightarrow+\infty$. Obviously, $u_{0}(t) \neq 0$; otherwise, $e(t)=0$, which contradicts condition $\left[H_{2}\right]$. This completes the proof.

\section{Acknowledgements}

The authors express their thanks to the referee for valuable suggestions.

\section{Funding}

This work was supported by National Natural Science Foundation of China under Grant No. 61803002, Natural Science Foundation of Anhui Province under Grant No. 1808085QF192, and Department of Education Scientific Research Foundation of Anhui Province under Grant No. KJ2018A0309.

\section{Competing interests}

The authors declare that they have no competing interests.

\section{Authors' contributions}

Both authors have equally contributed to obtaining new results in this paper and also read and approved the final manuscript.

\section{Author details}

${ }^{1}$ College of Physics and Electronic Information, Anhui Normal University, Wuhu, China. ${ }^{2}$ School of Automation, Nanjing University of Science \& Technology, Nanjing, China.

\section{Publisher's Note}

Springer Nature remains neutral with regard to jurisdictional claims in published maps and institutional affiliations.

Received: 29 March 2018 Accepted: 14 November 2018 Published online: 04 December 2018

\section{References}

1. Tang, X.H., Lin, X.Y.: Homoclinic solutions for a class of second-order Hamiltonian system. J. Math. Anal. Appl. 354, 539-549 (2009)

2. Tan, X.H., Li, X.: Homoclinic solutions for nonautonomous second-order Hamiltonian systems with a coercive potential. J. Math. Anal. Appl. 351, 586-594 (2009)

3. Zhang, Z.H., Yuan, R.: Homoclinic solutions for a class of non-autonomous subquadratic second-order Hamiltonian systems. Nonlinear Anal. 71, 4125-4130 (2009)

4. Lu, S.P., Lu, M.: Homoclinic solutions for a second-order $p$-Laplacian functional differential system with local condition. Adv. Differ. Equ. 2014, 244 (2014)

5. Zhang, X.Y., Zong, C., Deng, H.Y., Wang, L.B.: Existence and multiplicity of homoclinic solutions for difference systems involving classical $\left(\phi_{1}, \phi_{2}\right)$-Laplacian and a parameter. Adv. Differ. Equ. 2017, 380 (2017)

6. Su, Y.H., Yan, X.J., Jiang, D.H., Liu, F.H.: Homoclinic orbits for a class of second order dynamic equations on time scales via variational methods. Adv. Differ. Equ. 2017, 47 (2017)

7. Wu, D.L., Tang, C.L., Wu, X.P.: Homoclinic orbits for a class of second-order Hamiltonian systems with concave-convex nonlinearities. Electron. J. Qual. Theory Differ. Equ. 2018, 6 (2018)

8. Lu, S.P.: Existence of periodic solutions for neutral functional differential equations with nonlinear difference operator. Acta Math. Sin. Engl. Ser. 32, 1541-1556 (2016)

9. Lu, S.P., Zhang, L., Chen, L.J.: Homoclinic solutions for a class of second order neutral functional differential system. Acta Math. Sci. 33, 1361-1374 (2013)

10. Du, B.: Homoclinic solutions for a kind of neutral differential systems. Nonlinear Anal., Real World Appl. 13, 108-175 (2012)

11. Chen, W.B.: Homoclinic solutions for a class of neutral Duffing differential systems. Adv. Differ. Equ. 2014,121 (2014)

12. Tan, X.H., Li, X.: Homoclinic solutions for ordinary $p$-Laplacian systems with a coercive potential. Nonlinear Anal. TMA 71, 1124-1322 (2009)

13. Lu, S.P., Ge, W.G.: Sufficient conditions for the existence of periodic solutions to some second order differential equations with a deviating argument. J. Math. Anal. 308, 393-419 (2005)

14. Lu, S.P.: Homoclinic solutions for a class of second-order $p$-Laplacian differential systems with delay. Nonlinear Anal Real World Appl. 12,780-788 (2011) 
15. Lu, S.P.: On the existence of positive periodic solutions for neutral functional differential equation with multiple deviating arguments. J. Math. Anal. Appl. 280, 321-333 (2003)

16. Lu, S.P.: Periodic solutions to a second order $p$-Laplacian neutral functional differential system. Nonlinear Anal. 69 4215-4229 (2008)

Submit your manuscript to a SpringerOpen ${ }^{\circ}$ journal and benefit from:

- Convenient online submission

$\checkmark$ Rigorous peer review

Open access: articles freely available online

- High visibility within the field

- Retaining the copyright to your article

Submit your next manuscript at $\gg$ springeropen.com 